\title{
Formation of Black-Hole X-Ray Binaries with Low-Mass Donors
}

\author{
Vassiliki Kalogera \\ Harvard-Smithsonian Center for Astrophysics, Cambridge MA 02138, USA
}

\begin{abstract}
The characteristics of black-hole X-ray binaries can be used to obtain information about their evolutionary history and the process of black-hole formation. In this paper I focus on systems with donor masses lower than the inferred black-hole masses. Current models for the evolution of hydrogen-rich, massive stars and of helium stars losing mass in a wind cannot explain the current sample of black-hole mass measurements. Assuming that the radial evolution of mass-losing massive stars is at least qualitatively accurate, I show that the properties of the BH companions lead to constraints on the masses of black-hole progenitors (at most twice the black-hole mass) and on the strength of winds in helium stars (fractional amount of mass lost smaller than about 50\%). Constraints on common-envelope evolution are also derived.
\end{abstract}

\section{Introduction}

Radial velocity measurements of the non-degenerate donors in X-ray binaries combined with information about donor spectra and optical light curves allow us to measure the masses of accreting compact objects [1]. At present, measured masses for nine X-ray transients exceed the optimum maximum neutron mass [2] and the binaries are thought to harbor black holes (BH). Studies of the properties of such systems can shed a light to their evolutionary history and the process of BH formation.

Black-hole X-ray transients correspond to low-mass X-ray binaries with neutron stars (NS) in that mass transfer is driven by Roche-lobe overflow and the donors are less massive than the $\mathrm{BH}$. This critical (maximum) mass ratio of about unity allows the donor to transfer mass stably to the compact object. However, for the majority of the BH X-ray transients (including six $\mathrm{BH}$ candidate systems based on their spectral properties [3]) the donors are less massive than $\sim 1 \mathrm{M}_{\odot}$, much less massive than the typical $\mathrm{BH}$ masses. Only two systems, J1655-40 and 4U1543-47, have donors of $\sim 2.3 \mathrm{M}_{\odot}$ and $\sim 2-3 \mathrm{M}_{\odot}$, respectively. This apparent paucity of intermediate-mass donors (more massive than $\sim 2 \mathrm{M}_{\odot}$ ) cannot be explained by mass-transfer stability considerations alone.

In what follows, we address the issue of donor masses in $\mathrm{BH}$ binaries by studying a larger set of constraints imposed on their progenitors. We find that there is a strong dichotomy between the formation of systems with lowand intermediate-mass donors. Formation of both at the appropriate relative 
fraction requires little mass loss at $\mathrm{BH}$ formation, weak helium-star winds, and possibly energy sources other than the orbit for common-envelope (CE) ejection. This study and the results obtained are described in more detail in 顿.

\section{Evolutionary Constraints}

We consider BH X-ray binary formation from primordial binaries with extreme mass ratios evolving through a $\mathrm{CE}$ phase, similar to the formation channel for NS low-mass X-ray binaries, e.g., [5]. The primary must be massive enough so that its helium core exposed at the end of the $\mathrm{CE}$ phase collapses into a $\mathrm{BH}$. The X-ray phase is initiated when the donor fills its Roche lobe because of orbital shrinkage through magnetic braking (for lowmass donors) or of radial expansion through nuclear evolution on the main sequence (for intermediate-mass donors).

Black-hole binary progenitors evolve through this path provided that the following constraints are satisfied:

- The orbit is small enough that the primary fills its Roche lobe and the binary enters a CE phase.

- At the end of the CE phase the orbit is wide enough so that both the helium-rich primary and its companion fit within their Roche lobes. The constraint for the companion turns out to be stricter.

- The system remains bound after the collapse of the helium star. In the absence of kicks imparted to the $\mathrm{BH}$, this sets an upper limit on the mass of the $\mathrm{BH}$ progenitor.

- After the collapse, the orbit must be small enough so that mass transfer from the donor starts before it leaves the main sequence and within $10^{10} \mathrm{yr}$.

- Mass transfer from the donor proceeds stably and at sub-Eddington rates. This sets an upper limit to the donor mass on the zero-age main sequence and to the orbital size for more evolved donors.

\section{Donor Masses in Black-Hole X-ray Binaries}

For a specific value of the $\mathrm{BH}$ mass, the above constraints translate into limits on the properties, circularized post-collapsed orbital sizes $(A)$ and donor masses $\left(M_{d}\right)$, of $\mathrm{BH}$ binaries with Roche-lobe filling donors. The relative positions of these limits on the $A-M_{d}$ plane and the resulting allowed $M_{d}$ ranges are exactly determined by three well constrained model parameters:

- The amount of mass loss from the binary during BH formation, characterized by the ratio $M_{H e, f} / M_{\mathrm{BH}}$, where $M_{H e, f}$ is the mass of the helium-rich $\mathrm{BH}$ progenitor at the time of the collapse. For the post-collapse system to remain bound it must be $1 \leq M_{H e, f} / M_{\mathrm{BH}} \leq 3$. 
- The amount of mass lost in the helium-star wind between the end of the $\mathrm{CE}$ phase and the $\mathrm{BH}$ formation, characterized by the ratio $M_{H e, f} / M_{H e}$, where $M_{H e}$ is the initial helium-star mass (at the end of the CE phase). This ratio must lie in the range $0-1$.

- The CE efficiency, $\alpha_{C E}$, defined as the ratio of the CE binding energy to the orbital energy released during the spiral-in of the companion. Although the absolute normalization of $\alpha_{C E}$ is not well determined [ 4 , values higher than unity imply the existence of energy sources other than the orbit (ionization or nuclear burning energy).

Note that the last two of the constraints depend only on the BH mass, while $\alpha_{C E}$ affects only the upper limit on $A$ (first of the constraints $\S 2$ ). For different values of these three parameters, the positions of the limits on the $A-M_{d}$ plane change and three different outcomes with respect to the donor masses are possible: $\mathrm{BH}$ binaries can be formed with (i) only low-mass; (ii) only intermediate-mass; (iii) both low- and intermediate-mass donors.
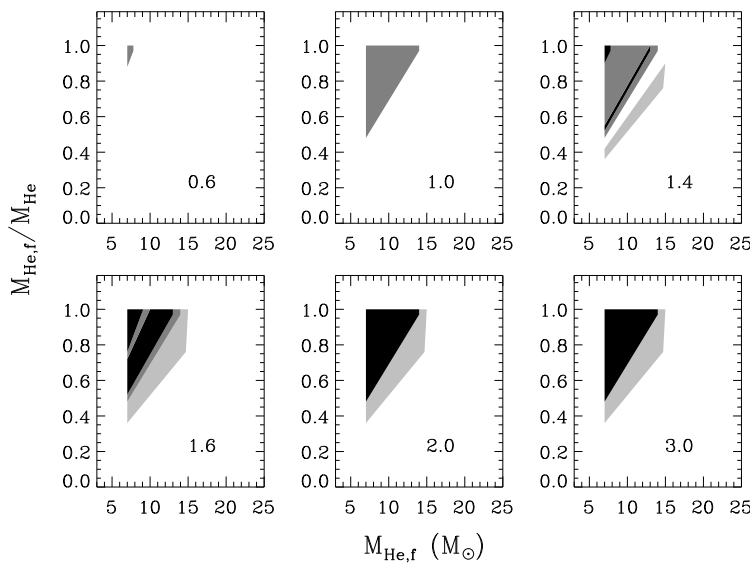

Fig. 1. Limits on the parameter space of the final (pre-collapse) helium-star mass, $M_{H e, f}$, and the ratio, $M_{H e, f} / M_{H e}$, for six values of the $\alpha_{\mathrm{CE}}=0.6,1.0,1.4,1.6$, 2.0, 3.0, and for a $7 \mathrm{M}_{\odot} \mathrm{BH}$. Conditions in the unshaded areas do not allow the formation of $\mathrm{BH}$ binaries with main-sequence Roche-lobe filling donors; conditions in the light-gray, dark-gray, and black areas allow the formation of systems with only low-mass, only intermediate-mass, and both types of donors, respectively.

The donor types as a function of the three parameters, $M_{H e, f}, M_{H e, f} / M_{H e}$, and $\alpha_{\mathrm{CE}}$, are shown in Fig. 1 , for a $7 \mathrm{M}_{\odot}$ BH. For $\alpha_{\mathrm{CE}}$ smaller than $\sim 0.5$, the orbital contraction is so high that the donor stars cannot fit in the post-CE orbits, and hence no $\mathrm{BH}$ X-ray binaries are formed. As $\alpha_{\mathrm{CE}}$ increases, $\mathrm{CE}$ ejection without the need of strong orbital contraction becomes possible for the more massive of the donors, while formation of binaries with low-mass 
donors occurs only if $\alpha_{\mathrm{CE}}>1.5$. The results become independent of $\alpha_{\mathrm{CE}}$ for values in excess of $\sim 2$, when the upper limit for $\mathrm{CE}$ evolution (first of the constraints) lies at high enough values of $A$ that it never interferes with the other limits.

The dependence of these results on the two mass-loss parameters (wind anf collapse) are determined by their association with orbital expansion. For strong helium-star wind mass loss $\left(M_{H e, f} / M_{H e}<0.35\right)$, the progenitor orbits expand so much that donors less massive than the $\mathrm{BH}$ can never fill their Roche lobes on the main sequence. Both low- and intermediate-mass donors are formed only if less than $50 \%$ of the initial helium-star mass is lost in the wind. Mass loss at $\mathrm{BH}$ formation is limited to $\mathrm{BH}$ progenitors less massive than about twice the $\mathrm{BH}$ mass so that post-collapse systems with low-mass donors remain bound.

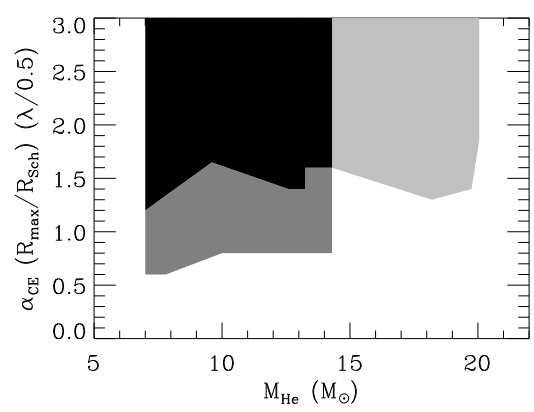

Fig. 2. Limits on the parameter space of the initial (post-CE) helium-star mass, $M_{H e}$, and the common-envelope efficiency, $\alpha_{\mathrm{CE}}$, properly normalized (by the maximum stellar radii of massive stars [6] and the central-concentration parameter, $\lambda$ ), for a $7 \mathrm{M}_{\odot}$ BH. Shade coding is as in Figure 1.

The dependence on $M_{H e, f}$ of the orbital expansion during helium-star wind mass loss and $\mathrm{BH}$ formation is such that the ratio of circularized postcollapse over post-CE orbital separations becomes independent of $M_{H e, f}$. This means that, for a specific BH mass, the position of the limits on the $A-M_{d}$ plane depend only on the initial helium-star mass and the CE efficiency. Indeed, in Fig. 1, the change of donor types occurs along straight lines in the $M_{H e, f} / M_{H e}-M_{H e, f}$ plane, or else along lines of constant $M_{H e}$. This simplifying property allows us to combine the panels in Fig. 1 into one plot (Fig. 2). It is evident that formation of $7 \mathrm{M}_{\odot} \mathrm{BH} \mathrm{X}$-ray binaries with both low- and intermediate-mass donors (as required by the observed sample) constrains the common-envelope efficiency to relatively high values and the initial helium-star progenitors at most twice as massive as the $\mathrm{BH}$ (corresponding to initial primaries in the range $25-45 \mathrm{M}_{\odot}$ ).

Additional constraints can be obtained by examining the relative numbers of systems with low- and intermediate-mass donors formed for the parameters 
in the black-shaded areas in Figs. 1 and 2. The lifetimes for the two different types are determined by the process that drives mass transfer. The magneticbraking timescale, for low-mass donors is comparable to the nuclear evolution timescale of intermediate-mass stars [1]. The number ratio then becomes equal to the ratio of birth rates. The latter can be calculated using the derived limits on $A$ and $M_{d}$ and assumed distributions of mass ratios and orbital separations of primordial binaries. The results indicate that even when lowmass companions in primordial binaries are strongly favored, $\mathrm{BH}$ binaries with intermediate-mass donors are much more easily formed because of the larger range of orbital separations allowed to their progenitors. Models predict a paucity of intermediate-mass donors only for rather high $\alpha_{\mathrm{CE}}$ values $(>3)$ or for moderate $\alpha_{\mathrm{CE}}$ values $(1.5-2)$ and $\mathrm{BH}$ progenitors either slightly more massive or twice as massive as the $\mathrm{BH}$.

\section{Discussion}

We find that the models for $\mathrm{BH}$ formation are consistent with the properties of the observed sample if (i) wind mass-loss from helium stars is limited so that they lose less than half of their initial mass (ii) helium stars that form black holes are at most twice more massive than the black holes, and (iii) $\mathrm{CE}$ efficiencies are relatively high and, depending on the exact radii of massive stars and their density profiles, significant contributions from energy sources other than the orbit may be required. Note that amounts of mass lost in helium-star winds and in BH formation are actually anti-correlated. If one is close to the maximum allowed then the other must be minimal (see Fig. 1). These results are quite robust and do not depend on the assumed BH mass nor the properties of primordial binaries.

The present study allows us to place constraints on the extent of wind mass loss from helium stars as they evolve towards collapse, primarily because helium cores are exposed at the end of the CE phase prior to core helium exhaustion. Current models of helium-star evolution through core helium burning [7] predict amounts of mass lost in the wind significantly larger than the maxima allowed for $\mathrm{BH}$ X-ray transient formation $(<50 \%)$. In fact, the final helium-star masses in these models are $\sim 4 \mathrm{M}_{\odot}$, far too small to explain the $\mathrm{BH}$ mass measurements. Therefore, if the $\mathrm{CE}$ phase is initiated early in the core helium burning phase of the primary, then helium-star winds must be much weaker than thought until now. It is noteworthy that more recent empirical estimates of wind mass loss rates [8] show a downward trend.

The strength of helium-star winds becomes irrelevant to the process of $\mathrm{BH} \mathrm{X}$-ray binary formation, if the $\mathrm{CE}$ phase is initiated late in the evolution of the massive primary, i.e., after core helium exhaustion. In this case the helium star is exposed only through carbon burning and later evolutionary phases. The total duration of these phases is so short that the wind mass loss is insignificant and the helium-star mass remains essentially constant [7]. 
Current models of massive star evolution [6], though, permit CE evolution at such late stages only for primary masses lower than $\sim 25 \mathrm{M}_{\odot}$ and for an extremely narrow range of orbital separations [9]. For more massive stars, there is not enough radial expansion (in fact the radius decreases) to counterbalance the orbital expansion due to wind mass loss from the hydrogen-rich primary during core helium burning, and bring the primary to Roche-loe overflow. Therefore, relying on $\mathrm{CE}$ evolution occurring only at late stages cannot account for $\sim 10 \mathrm{M}_{\odot} \mathrm{BH}$ and it is possible only for a tiny fraction of primordial binaries leading to uncomfortably low birth rates for BH X-ray binaries [10]. All these problems can be circumvented only if the radial evolution predicted by the current models of mass-losing massive star evolution is incorrect both qualitatively and quantitatively and stars more massive than $\sim 25 \mathrm{M}_{\odot}$ expand significantly after core helium exhaustion. Such a modification of the massive-star models has been assumed by Wellstein \& Langer [11] and no reduction of helium-star wind mass loss rates is then required. Given the uncertainties in models of massive star evolution such a modification cannot be excluded at present. In any case, it becomes clear that the existence of X-ray transients with $\sim 10 \mathrm{M}_{\odot} \mathrm{BH}$ requires that either the hydrogen-rich massive star models or the strength of helium-star winds be modified.

Acknowledgments. I would like to thank N. Langer and S. Wellstein for useful discussions. Support by the Smithsonian Astrophysical Observatory through a Harvard-Smithsonian Center for Astrophysics Postdoctoral Fellowship is also acknowledged.

\section{References}

1. Charles, P.A. 1998, in Theory of Black Hole Accretion Disks, eds. M.A. Abramowicz, G. Bjornsson \& J.E. Pringle, (Cambridge: Cambridge University Press), 1

2. Kalogera, V. \& Baym, G. 1996, ApJ, 470, L61

3. Chen, W., Shrader, C.R., \& Livio, M. 1997, ApJ, 491, 312

4. Kalogera, V. 1999, ApJ, 521, 723

5. van den Heuvel, E.P.J. 1983, in Accretion-Driven Stellar X-Ray Sources, ed. W. H. G. Lewin, \& E. P. J. van den Heuvel (Cambridge: Cambridge University Press), 303

6. Schaller, G., Schaerer, D., Meynet, G., \& Maeder, A. 1992, A\&A Suppl. Series, 269,331

7. Woosley, S.E., Langer, N., \& Weaver, T.A. 1995, ApJ, 448, 315

8. Hamann, W.-R. \& Koesterke, L. 1998, A\&A, 335, 1003

9. Kalogera, V. \& Webbink, R.F. 1998, ApJ, 493, 351

10. Portegies-Zwart, S.P., Verbunt, F., \& Ergma, E. 1997, A\&A, 321, 207

11. Wellstein, S. \& Langer, N. 1999, A\&A, 350, 148 